\title{
CATECHOLAMINE SECRETING TUMORS - AN ANESTHETIC
}

\section{CHALLENGE}

Authors: Carreteiro J., Alves D., Costa Martins I., André A.I., Palma Mira F., Aguiar I.

Institute: Centro Hospitalar de Lisboa Ocidental, E.P.E.

\section{BACKGROUND}

Catecholamine-secreting tumors present particular challenges to anaesthesiologists ${ }^{1}$. Intraoperative success relates to proper preoperative optimization, which should be ensured. ${ }^{1}$

\section{CASE REPORT}

\section{Case 1}

Female, 45y with paraspinal mass invading T1-T2 $\rightarrow$ proposed for tumor excision. Intraoperatively: mass mobilization associated with marked haemodynamic instability
Admitted to ICU and a functioning paraganglioma was diagnosed

$$
\downarrow
$$

Stabilization and 9-day course of $\alpha$ and $\beta$ blockers
Angiographic embolization of the mass followed by new surgical intervention: initial mobilization of the tumor was still accompanied by adrenergic manifestations

\section{Case 2}

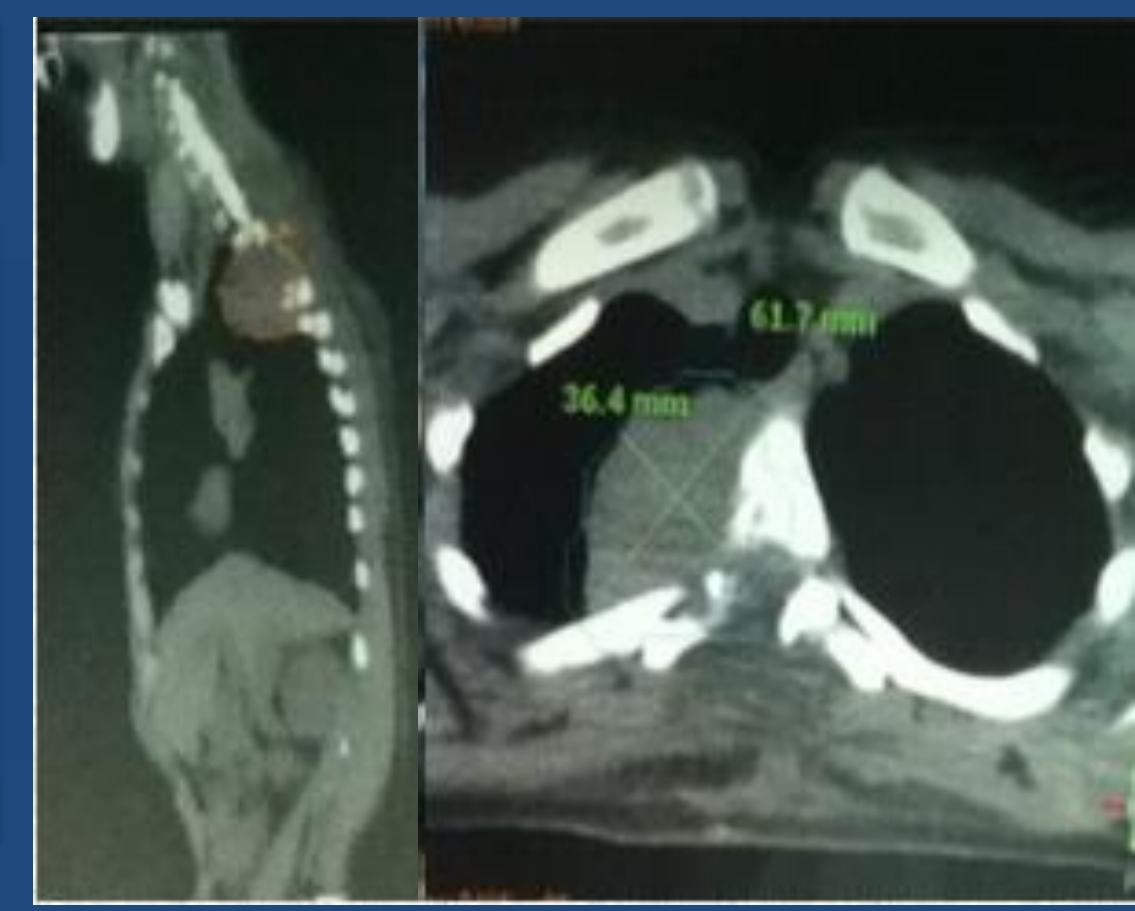

Figure 1 - CT scan of Case 1 showing thoracic paraganglioma

Preoperatively: 30 -day

Female, $64 y$ with repeated syncope, paroxysmal tachycardia and resistant hypertension.

Diagnosis: suprarenal mass suggestive of pheochromocytoma therapy with $\alpha$ and $\beta$ blockers

$\rightarrow$ Surgical procedure progressed uneventful

A week later symptoms recurred $\rightarrow$ study revealed a functioning paranganglioma
Second intraoperative period went with marked haemodynamic instability aggravated by accidental laceration of the left renal vein

\section{Case 3}

Male, 26 y $\rightarrow$ adrenalectomy for pheochromocytoma excision
Preoperatively: 21 days of $\alpha$ blocker therapy for hemodynamic stabilization
Intraoperatively: isolated hypertensive peak during surgical manipulation of the tumor

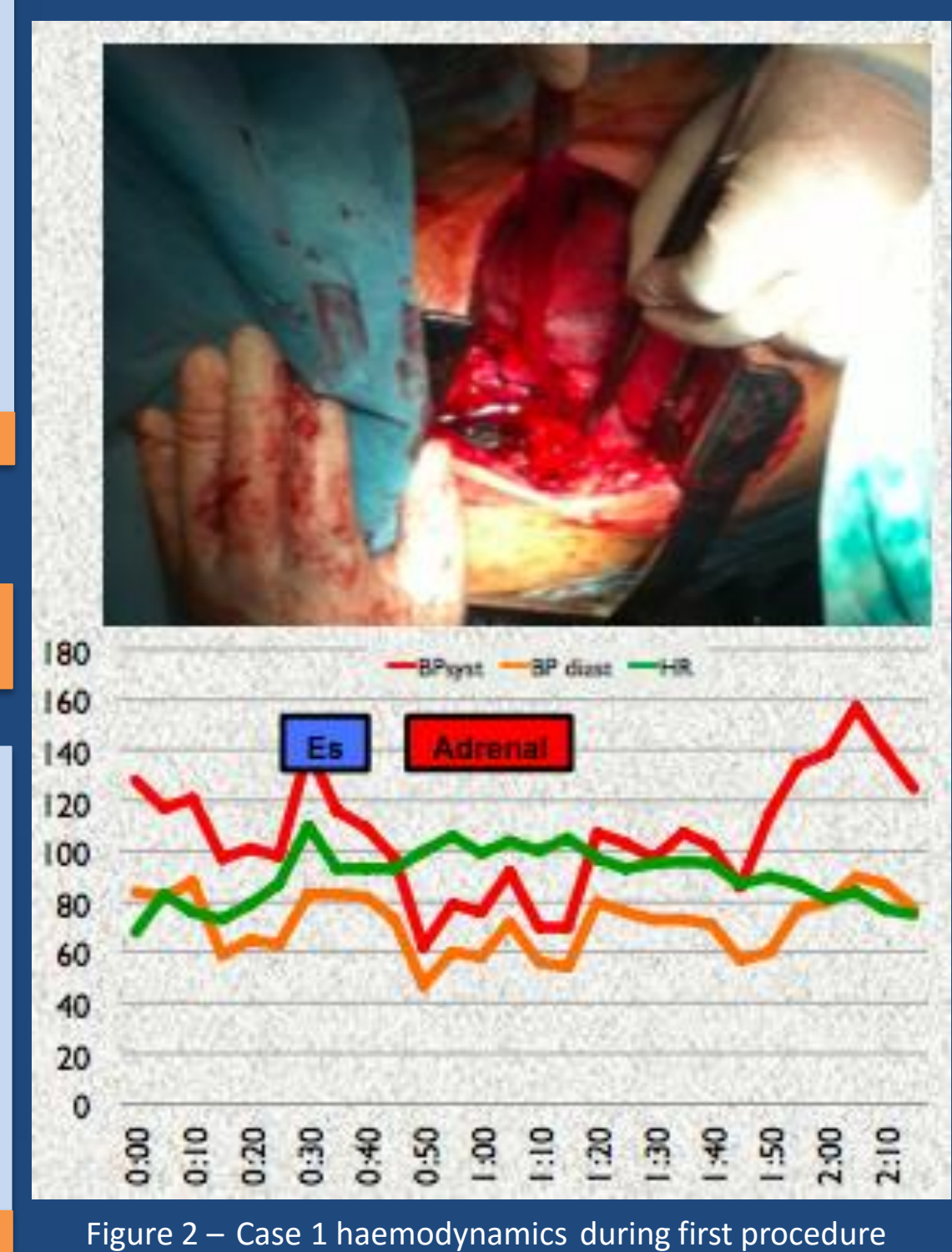

\section{DISCUSSION}

There must be a high degree of suspicion towards posterior mediastinum and periadrenal masses as clinical presentation can be atypical ${ }^{2}$. Furthermore, intraoperative mortality associated with catecholamine-secreting tumors not previously identified reaches $50 \%$, being reduced to less than $2 \%$ with adequate preoperative optimization.

All patients developed hypotension after tumor isolation from circulation, requiring vasopressors whose effectiveness allowed an uneventful post-operative ICU period.

\section{LEARNING POINTS}

Correct preoperative stabilization and anticipation of intraoperative problems with low threshold for intervention prove to be essential to a favorable outcome in such tumors. 